\section{Concerns, challenges and promises of high-content analysis of 3D cellular models}

\author{
Neil Carragher, Filippo Piccinini, Anna Tesei, O. Joseph Trask Jr, Marc Bickle and \\ Peter Horvath
}

There is intense excitement in the scientific community about 3D cellular model systems because they promise to resemble and recapitulate the in vivo tissue environment more faithfully than $2 \mathrm{D}$ systems ${ }^{1}$. A rapidly expanding offering of commercially available in vitro technologies for high-throughput 3D cellbased disease models, combined with advances in material sciences, are enabling widespread application and adoption of these models across academic and industrial research groups. However, there is a lack of conclusive evidence that such models accurately recapitulate in vivo tissue physiology and disease pathophysiology, and thereby provide sufficiently quantitative and reproducible data to replace current models and improve the clinical success rates of drug candidates. Prominent cellular high-content screening (HCS) and bioimage informatics societies are therefore calling for further debate to discuss the value of these emerging 3D model systems in an effort to establish more transparent and standardized guidelines in the field. Specifically, scientific community representatives highlight the lack of validated methodologies and software tools that enable robust quantitative analysis of the vast number of newer 3D cellular models.
With this in mind, we conducted a SWOT (strengths, weaknesses, opportunities and threats) analysis of the pipeline for highcontent analysis of 3D cellular systems, divided into the four main stages: system development, imaging, screening and data analysis. Here, we summarize the main outcomes of this SWOT analysis, which are highlighted in FIG. 1. Each point is further discussed in more depth and references are provided in Supplementary Box 1.

\section{SWOT analysis}

There have been substantial developments in the field of cell-based screening during the last decade, such as the emergence of stem cell technologies, microtissues, organoid models and organ-on-a-chip platforms. Relevant differences in cellular behaviour between 2D and $3 \mathrm{D}$ cultures have been characterized in several prominent studies, reporting the greater physiological relevance of 3D models compared with similar 2D models. In most cases, cells are grown embedded in extracellular matrices to form organoids or spheroid-like structures. However, these models do not
- Phenotypic cell differentiation

- Physiological relevance

- Patient-derived models

- Stem cell models

- Animal model substitute

- Biological heterogeneity

- Target validation

\section{o}

- Orthogonal cell type interactions

- Ex vivo clinical trials

- Fluidic systems

- Manipulate the extracellular

matrix

System development

W

- Morphological heterogeneity - Size and shape-based viability - Spatial dependence of physiological state - Simplified organ models

- Validation uncertain

- No vascularization system

- Dynamic tuning of tumour ecosystem - Long, complicated cell culture protocols

\section{- Expensive}

- Challenging model validation

- Current lack of clinical validation

- Incentives and funding
S

- Current equipment is sufficient

- Clearing protocols exist

- In vivo diagnostic technology analogy

\section{o}

- Optical physics technology innovations

- Customized supports

- Development of photostable

fluorophores
Imaging

- Limited penetration depth - Limited holder solutions

- Requirement for object search

- Bleaching of fluorophores

- Multiscale imaging requirement
Data analysis

- New information-rich data sets

- Spatial organization analysis

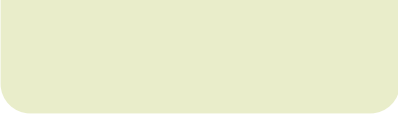

O

- Exploit computational biology methods

- Accessible low-cost technology innovations w

-3D parameter understanding - Challenging volumetric analysis - Ease of use

\section{T}

- Limited know-how

- Computationally demanding

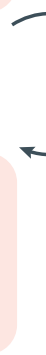

$\longrightarrow$

- High physiological relevance

- Available assays

- Novel 3D tissue models

- 4D in vitro pharmacology

O

- Intelligent screening

- Innovation in biological target space

- Innovation in pharmacological target space

- Clinical-like conditions
Screening

Needs optimized equipment

- Image quality

- Long acquisition times

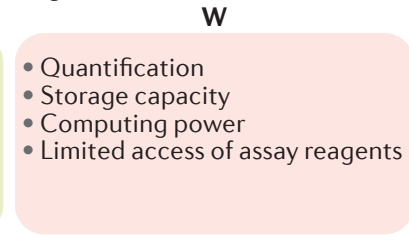

T

- Speed limit

- Expensive

- Low adoption

- In vitro to in vivo translation

- Data reproducibility

Figure 1 | SWOT analysis of the four major steps in high-content screening using 3D cellular systems. For an expanded list of points with references, please see Supplementary Box 1. SWOT, strengths, weaknesses, opportunities and threats. 
fully represent the complexity of many in vivo tissues, often lacking relevant cell types and required growth factors, and are not subject to long-distance signalling from other organs, the immune system, the endocrine system or the microbiome. In particular, most current models are static and do not model the dynamics of in vivo tissue perfusion and mechanical stress forces. Emerging microfluidic and macrofluidic technologies that simulate a circulatory system allow perfusion of 3D tissues, and can induce shearing forces while permitting the observation of the microtissue at the same time. However, the majority of systems so far have not been designed for automated screening applications and currently there is no off-the-shelf solution compatible with conventional HCS platforms. One of the next challenges will therefore be miniaturizing these systems in order to screen hundreds to thousands of microtissues at the same time in a cost-effective manner.

At present, there is still a lack of both qualitative and quantitative evidence demonstrating that such 3D model systems better predict clinical response relative to existing in vivo approaches. So, although the advantages of 3D model systems appear to be clear, we caution not to overestimate their physiological relevance given their relative simplicity in comparison to in vivo tissues. We also strongly encourage further development, validation, and investment in comparative phenotypic, genomic, proteomic and metabolomic analysis between new 3D model systems with both healthy and diseased human tissues.

Imaging of 3D model systems poses substantial challenges owing to light scattering within the objects and the non-uniform positioning of objects in microplate wells. These challenges can be partially addressed with smart techniques on automated microscopes and chemical clearing protocols to allow deeper penetration of light into tissue, but many areas require improvement, especially if 3D imaging is to be applied in HCS.

Given the vast amount of data generated in a HCS campaign, every byte is precious and therefore every pixel has to be of the highest quality. Importantly, given the need for extensive functional and mechanistic studies, the need to develop better imaging techniques and the vast amount of data produced in screens, our analysis highlights the lack of user-friendly analytical tools. Opensource algorithms for 3D image analysis, visualization and statistical analysis exist, but they are not accessible to biologists and require extensive computational expertise. This represents a major hurdle for the adoption, development and validation of advanced 3D model systems (see Supplementary Box 1 for more detail).

\section{Conclusion}

We believe there is a need for more early multidisciplinary collaborations, methodology guidelines, better communication and education in this field to provide the community with a more transparent assessment of the value of new 3D models and a customized toolkit for the users of 3D technologies. In addition, we call for collaborative 3D cellular model development between industry and academia to promote the adoption of standardized 3D assay technologies.

We also wish to emphasize that it is not beneficial for the field to oversell currently available models because they have neither been clinically validated nor are they likely complex enough to mimic in vivo physiology faithfully. We have often witnessed an early enthusiasm for technologies followed by disillusionment due to inflated expectations and disappointing results. In retrospect, it was clear that the technology was not mature and needed further research and development in order to better understand its shortcomings and strengths. Similarly, with 3D cell culture systems, we run the risk of disillusionment if we do not establish appropriate guidelines on how to validate a model, how to cultivate the cells, how to acquire the image data and how to analyse the resulting data. Nevertheless, with awareness of their limitations and concerted efforts to close the gaps in validating the relevance of 3D cell model systems and associated technologies, such systems may enable important advances in drug discovery and fundamental biology.

Neil Carragher is at the Cancer Research UK Edinburgh Centre, MRC Institute of Genetics and Molecular Medicine, University of Edinburgh, Edinburgh, UK.

Filippo Piccinini and Anna Tesei are at the Istituto Scientifico Romagnolo per lo Studio e la Cura dei Tumori (IRST) IRCCS, Meldola (FC), Italy. O. Joseph Trask, Jr is at PerkinElmer, Inc., Waltham, MA, USA.

Marc Bickle is at the Max Planck Institute of Molecular Cell Biology and Genetics, Dresden, Germany.

Peter Horvath* is at the Biological Research Centre of the Hungarian Academy of Sciences, Szeged, Hungary and the Institute for Molecular Medicine Finland (FIMM), University of Helsinki, Helsinki, Finland.

*e-mail: horvath.peter@brc.mta.hu doi:10.1038/nrd.2018.99

Published online 6 Jul 2018

1. Kingwell, K. 3D cell technologies head to the $R \& D$ assembly line. Nat. Rev. Drug Discov. 16, 6-7 (2017).

Acknowledgements

P.H. acknowledges support from the LENDULET-BIOMAG Grant (2018-342) and support from the European Regional Development Funds (GINOP-2.3.2-15-2016-00001,

GINOP-2.3.2-15-2016-00026). F.P. acknowledges support from the NEUBIAS, COST Action (European Cooperation in Science and Technology) CA15124, for a granted short term scientific mission (ref: 41037)

\section{Competing interests}

The authors declare competing interests: see Web version for details.

Supplementary information

Supplementary information is available for this paper at http://www.nature.com/articles/nrd.2018.99. 